\title{
Plasma Catecholamines Assay: \\ Comparison Between Fluorimetric and Radioenzymatic Methods ${ }^{1}$ )
}

\author{
By E. Agabiti-Rosei, M. Beschi, M. Castellano \\ Cattedra di Clinica Medica Generale e Terapia Medica, Università degli Studi di Brescia, Brescia (Italia)
}

\section{S. Tosoni and C. Signorini}

Cattedra di Chimica e Propedeutica Biochimica, Università degli Studi di Brescia, Brescia (Italia)

(Received November 11, 1983/March 14, 1984)

Summary: Plasma catecholamines were simultaneously measured in duplicate plasma samples by the fluorimetric method of Renzini et al. ((1970) Clin. Chim. Acta 39, 587-594) and by the radioenzymatic method of Da Prada \& Zurcher ((1976) Life Sci. 19, 1161-1174).

The correlation of noradrenaline and adrenaline plasma concentrations determined by the two methods were, respectively, $r=0.95(p<0.001)$ and $r=0.75(p<0.01)$. The fluorimetric method was less sensitive, but more economical and less time-consuming than the radioenzymatic method. The fluorimetric method is still of value for measuring plasma catecholamines in man.

\section{Bestimmung der Catecholamine im Plasma:}

Vergleich zwischen fluorimetrischer und radioenzymatischer Methode

Zusammenfassung: Die Catecholamine im Plasma wurden in Doppelbestimmungen gleichzeitig mit der fluorimetrischen Methode von Renzini et al. ((1979) Clin. Chim. Acta 39, 587-594) und der radioenzymatischen Methode von Da Prada \& Zurcher ((1976) Life Sci. 19, 1161-1174) bestimmt.

Die Korrelation der mit beiden Methoden bestimmten Konzentrationen betrug $r=0,95(p<0,001)$ für Noradrenalin und $r=0,75$ ( $p<0,01$ ) für Adrenalin. Die fluorimetrische Methode war weniger empfindlich, jedoch ökonomischer und weniger zeitraubend als die radioenzymatische. Die fluorimetrische Methode ist noch immer von Bedeutung für die Bestimmung der Catecholamine beim Menschen.

\section{Introduction}

The adrenergic nervous system has a major role in the regulation of circulation and metãbolism in several pathophysiological conditions (1). At the present time, plasma catecholamines are probably the best index for measuring adrenergic activity in man, as no electrophysiological or other methods are readily available for this purpose $(2,3)$. The recent

\footnotetext{
1) This work was supported in part by grant No. 82.02249.56 of the National Research Council - Rome - Italy.
}

introduction of sensitive and specific techniques for measuring plasma noradrenaline and adrenaline has therefore aroused increased interest (4-9). Only a few studies, however, have reported a direct comparison between the available methods $(10,11)$.

In this study we have compared the sensitive and specific fluorimetric technique of Renzini et al. (5) with the more modern radioenzymatic technique of Da Prada \& Zurcher (7). To our knowledge such a direct comparison has not been previously performed. 


\section{Materials and Methods}

Apparatus

The following instruments were used: Liquid scintillation spectrometer Tri-Carb model 3385, supplied by Packard Instrument Company, Inc., Downers Grove, IL 60515, USA; Centrifuges Model J-6 and Model J-21C supplied by Beckman Instruments, Geneva, Switzerland; Aminco-Bowman spectrophotofluorimeter supplied by American Instrument Company, Silver Spring, Maryland, USA.

\section{Reagents}

Reagents and their sources were as follows: dithiothreitol, metanephrine- $\mathrm{HCl}$, normetanephrine- $\mathrm{HCl}$, ethyleneglycol-bis(amino-ethyl ether)-N,N'-tetraacetic acid, tris(hydroxymethyl) aminomethane base, tetraphenylborate and 2,3-dimercaptopropanol from Sigma Chemical Co., St. Louis, MO 63178, USA; aluminium oxide active neutral, Amberlite CG- $50 \mathrm{H}, L$-adrenaline and $L$-noradrenaline from $\mathrm{BDH}$ Chemicals Ltd, Poole, Dorset, U.K.; Kieselgel LK6F, $20 \times 20 \mathrm{~cm}$ plates from Whatman Inc., Clifton, NJ 07014, USA; [ ${ }^{3} \mathrm{H}$-methyl]S-adenosyl- $L$-methionine of specific activity $40.54 \mathrm{GBq} / \mathrm{mol}$ from Amersham International Ltd., Amersham, Bucks., U.K.; scintillation cocktail Liquiflor from New England Nuclear Corp., Boston, MA 02118, USA. All solvents were of analytically pure grade and were supplied by Merck, Darmstadt, F.R.G.; the other reagents were supplied by commercial sources.

The enzyme catechol-O-methyltransferase was extracted from rat liver according to the procedure of Axelrod \& Tomchick (12).

Catecholamine stock standard solutions were prepared by dissolving adrenaline and noradrenaline in $\mathrm{HCl} 0.01 \mathrm{~mol} / \mathrm{l}$ to obtain a concentration of $10 \mathrm{mg}$ of catecholamine base per liter. These solutions were stored in a dark bottle at $4{ }^{\circ} \mathrm{C}$, under which conditions they are stable for at least 6 months.

All the reagents were stored at $4^{\circ} \mathrm{C}$, except catechol-O-methyltransferase and $\left[{ }^{3} \mathrm{H}\right.$-methyl $] \mathrm{S}$-adenosyl- $L$-methionine, which were stored at $-20^{\circ} \mathrm{C}$ and $-80^{\circ} \mathrm{C}$, respectively.

\section{Sample collection}

Blood samples $(20-30 \mathrm{ml})$ were drawn from an antecubital vein into heparinized syringes, transferred immediately into ice-chilled polyethylene tubes and centrifuged, within $10 \mathrm{~min}$, at $5000 \mathrm{~min}^{-1}$ for $10 \mathrm{~min}$ at $4^{\circ} \mathrm{C}$. For the fluorimetric method $1 \mathrm{mg}$ of sodium metabisulphite was added for each milliliter of plasma; no antioxidant was added to the aliquots of samples collected for the radioenzymatic assay.

Plasma specimens were assayed with the two methods within 4 weeks of initial storage at $-30^{\circ} \mathrm{C}$. Plasma volumes were $8-10 \mathrm{ml}$ for the fluorimetric method and $0.1 \mathrm{ml}$ for the radioenzymatic method.

Plasma catecholamines assays

Plasma catecholamines were assayed simultaneously, using the radioenzymatic procedure of Da Prada \& Zurcher (7), and the fluorimetric method of Renzini et al. (5), without major modifications.

Principle of the radioenzymatic assay

This method is based on the 3-O-methylation of the catecholamines in the presence of the enzyme catechol-O-methyltransferase, with $\left[{ }^{3} \mathrm{H}\right.$-methyl]S-adenosyl- $L$-methionine as methyl donor. After methylation, the radioactively labeled products normetanephrine, metanephrine and methoxytyramine, are extracted into diethylether and separated by thin-layer chromatography. The labeled catecholamines are eluted, oxidized to vanillin and finally measured in a liquid scintillation counter.

\section{Principle of the fluorimetric assay}

This method is based on the separation and purification of adrenaline and noradrenaline achieved through a two-step chromatographic separation on alumina and amberlite CG-50 H columns, perchloric and boric acids being used for elution. Subsequently, fluorophors of high fluorescence intensity are formed by the trihydroxyindole reaction; lutine fluorescence is stabilized by using a solution of $10 \%$ dimercaptopropanol in $25 \%$ formaldehyde solution and making a final $\mathrm{pH}$ adjustment to about 5.3.

\section{Results}

\section{Specificity and sensitivity}

In agreement with Renzini et al., we found that noradrenaline and adrenaline show characteristic excitation spectra at an emission wavelength of $500 \mathrm{~nm}$. Plasma samples or external standards of noradrenaline and adrenaline have a major excitation peak at 415 and $430 \mathrm{~nm}$, respectively, with a minor peak at $305 \mathrm{~nm}$. These two distinct peaks allow sufficient precision for the separate calculations of noradrenaline and adrenaline concentrations. The radioenzymatic technique is highly specific, because the enzymatic reaction selectively methylates the catecholamines, and the chromatographic step permits the accurate discrimination of noradrenaline and adrenaline.

The smallest amounts of noradrenaline and adrenaline which show a fluorescence intensity capable of doubling the blank value are $0.24 \mathrm{pmol}$ and 0.22 pmol, respectively (with res̀pect to the amount present in the last oxidation step), or $1.77 \mathrm{pmol}$ and 1.64 pmol, respectively (with respect to the amount present in the plasma sample); this implies lower detection limits of $177 \mathrm{pmol} / \mathrm{l}$ plasma for noradrenaline and $164 \mathrm{pmol} / \mathrm{l}$ plasma for adrenaline. With the radioenzymatic technique the smallest amounts that can double the counts/min value of the blank are 0.015 pmol for noradrenaline and 0.011 pmol for adrenaline, per $100 \mu \mathrm{l}$ sample; the detection limits are therefore $150 \mathrm{pmol} / \mathrm{l}$ plasma for noradrenaline and $115 \mathrm{pmol} / \mathrm{l}$ plasma for adrenaline.

\section{Linearity}

There is a linear relationship between fluorescence intensity and catecholamine concentration in the range $0.5-100 \mathrm{pmol}$. This linearity has been verified for noradrenaline and adrenaline in pure solutions, and after adding known amounts of catecholamines to plasma, followed by the entire extraction and oxidation procedure. Considering the fluorescence in- 
tensity in arbitrary units at 415 and $430 \mathrm{~nm}$ for noradrenaline and adrenaline, respectively, the correlations were as follows:

Fluorescence intensity (units) $=$

0.04 pmol noradrenaline $+0.0695, r=0.9997$;

Fluorescence intensity (units) $=$

0.04 pmol adrenaline $+0.0128, r=0.9998$.

Similarly a linear relationship has been observed between catecholamine concentrations and counts/min using the radioenzymatic technique:

counts/min $=$

2.02 pmol noradrenaline $+0.594, r=0.9998$;

counts/min =

2.15 pmol adrenaline $+0.0225, \mathrm{r}=0.9989$.

The relationships between noradrenaline concentration and counts/min or fluorescence intensity are reported in figures 1 and 2.

\section{Reproducibility}

The reproducibility of both techniques for plasma catecholamine estimations has been evaluated by measuring aliquots of pooled plasma in duplicate in the same assay or in different assays. Intraassay mean variation coefficients for noradrenaline and

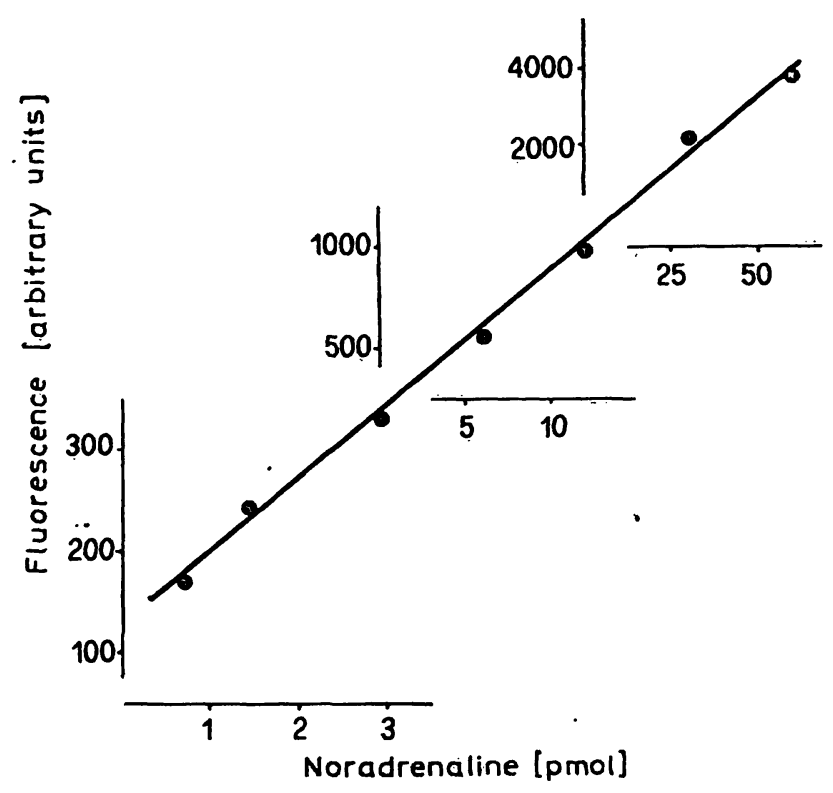

Fig. 1. Relationship between noradrenaline concentration and fluorescence intensity (arbitrary units).

Fluorescence intensity (units) $=0.04 \mathrm{pmol}$ noradrenaline $+0.0695, r=0.9997$.

Similar correlation was observed with adrenaline (see text). adrenaline are, respectively, $8 \%$ and $13 \%$ with the fluorimetric method, and $10 \%$ and $15 \%$ with the radioenzymatic method $(n=10$ duplicate samples for both techniques). Interassay variation coefficients for noradrenaline and adrenaline are, respectively, $14 \%$ and $20 \%$ with the fluorimetric method $(n=14$, noradrenaline concentration $2465 \pm 343 \mathrm{pmol} / \mathrm{l}$, adrenaline concentration $540 \pm 109 \mathrm{pmol} / \mathrm{l})$, and $12 \%$ and $18 \%$ with the radioenzymatic method $(n=16$, noradrenaline concentration $2205 \pm 266 \mathrm{pmol} / \mathrm{l}$, adrenaline concentration $704 \pm 126 \mathrm{pmol} / \mathrm{l})$.

Comparison of values determined by fluorimetry and by the radioenzymatic method

Figures 3 and 4 illustrate the relationship between the fluorimetric and enzymatic methods for the determination of plasma noradrenaline and adrenaline; in each case the catecholamines were determined simultaneously and in duplicate. A high correlation between the corresponding values of noradrenaline by these two methods was found in 25 plasma samples $(r=0.95$, noradrenaline fluor. $=1.044 \times$ noradrenaline radioenz. -0.0635 ). The correlation coefficient for adrenaline was lower, but still statistically significant $(r=0.75$, adrenaline fluor. $=0.8 \times$ adrenaline radioenz. +0.224$)$.

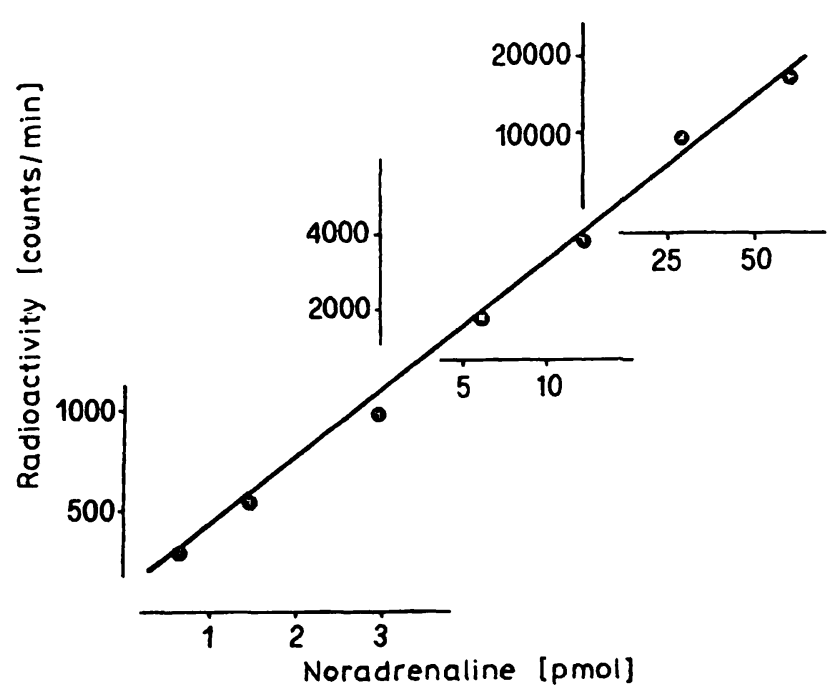

Fig. 2. Relationship between noradrenaline concentration and counts $/ \mathrm{min}$. Counts $/ \mathrm{min}=2.02 \mathrm{pmol}$ noradrenaline + $0.594, r=0.9998$.

Similar correlation was observed with adrenaline (see text). 


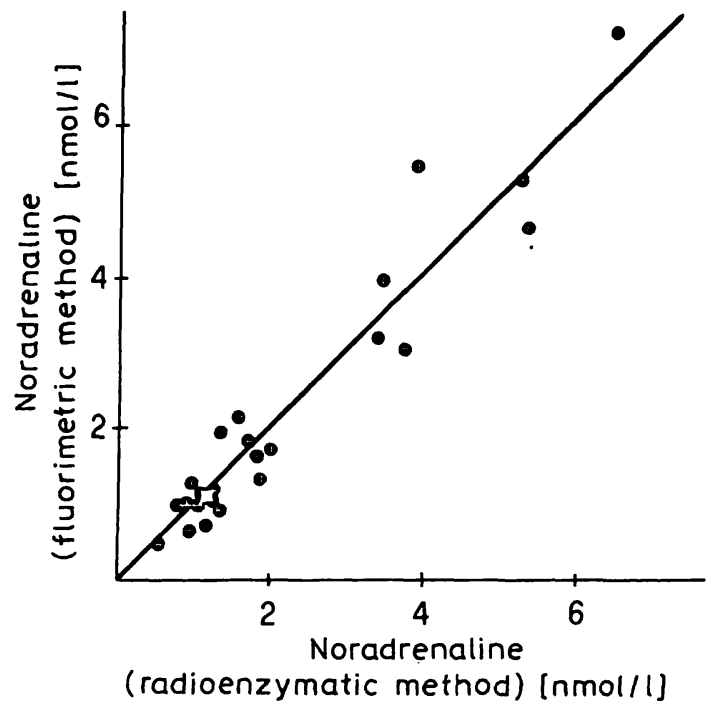

Fig. 3. Correlation of plasma noradrenaline concentrations determined in 25 duplicate plasma samples by the two assay methods.

Noradrenaline fluor. $=1.044 \times$ noradrenaline radioenz. $-0.0635, \mathrm{r}=0.95, \mathrm{p}<0.001$.

\section{Discussion}

In this study we demonstrated that the plasma concentration values of noradrenaline and adrenaline determined by both the fluorimetric and the radioenzymatic method are comparable over a wide range. The correlation coefficient was greater for noradrenaline than for adrenaline, and this was probably due to the fact that both methods, but mainly the fluorimetric, are not sensitive enough for measuring low plasma adrenaline concentrations (i.e. $<250 \mathrm{pmol} / \mathrm{l}$ ) accurately.

The radioenzymatic method has the advantage of being more sensitive than the fluorimetric method. On the other hand, the fluorimetric method is easier, more practical and inexpensive. In our experience, one technician can process 24 plasma specimens in 10-12 hours with the fluorimetric technique, and 10 plasma specimens in two working days with the radioenzymatic technique.

The fluorimetric technique is probably more convenient when only a few plasma samples ( 2 or 3 ) have to be collected from one patient. For repeated blood sampling in man or blood sampling in small animals, the radioenzymatic technique is more appropriate, because it requires only $1-2 \mathrm{ml}$ of blood.

Rigorously clean glassware and glass double distilled water are essential prerequisites for the fluorimetric

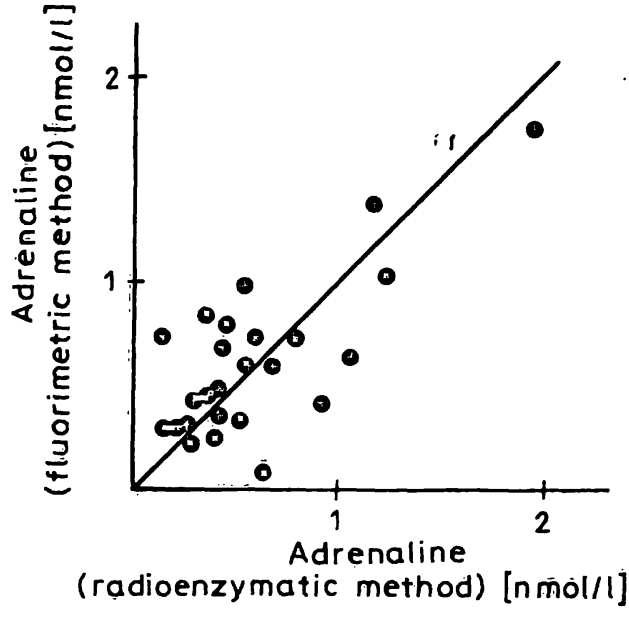

Fig. 4. Correlation of plasma adrenaline concentrations determined in 25 duplicate plasma samples by the two assay methods.

Adrenaline fluor. $=0.8 \times$ adrenaline radioenz. +0.224 , $r=0.75, p<0.001$.

technique. In addition, we tested reagents from various sources in order to find those with low and stable blank values.

To our knowledge this is the first study in which a direct comparison between the fluorimetric and the radioenzymatic methods has been performed. In a previous study by Miura et al. (11) the fluorimetric assay was compared to the older double-isotope technique of Engelman \& Portnoy (6), and the results obtained were similar to those observed in our study. Contrary to the observations of Miura et al. we found no advantage in substituting dimercaptopropanol with mercaptoethanol; actually, we found that the use of dimercaptopropanol gave greater sensitivity.

In conclusion, our findings demonstrate that the fluorimetric assay of Renzini et al. can be as reliable as the radioenzymatic technique of Da.Prada \& Zurcher for the measurements of plasma catecholamines in man. The reliability of this technique is greater for plasma noradrenaline than for plasma adrenaline in the lower concentration range.

\section{Acknowledgements}

The authors are grateful to Prof. Giulio Muiesan and Prof. Alberto Albertini for their valuable help and advice in revising the manu- script. 


\section{References}

1. Cryer, P. E. (1980) New Eng. J. Med. 303, 436-444.

2. Lake, C. R., Ziegler, M. G. \& Kopin, I. J. (1976) Life Sci. 18, $1315-1325$.

3. Agabiti-Rosei, E., Alicandri, C., Beschi, M., Castellano, M., Fariello, R., Muiesan, M. L., Romanelli, G. \& Muiesan, G. (1982) In: "Endocrinology of Hypertension" (Mantero, F., Biglieri, E. G. \& Edwards, C. R. W., eds.) Academic Press, London and New York, pp. 387-401.

4. Valori, C., Brunori, C. A., Renzini, V. \& Corea, L. (1970) Anal. Biochem. 33, 158-167.

5. Renzini, V., Brunori, C. A. \& Valori, C. (1970) Clin Chim. Acta 39, 587-594.

6. Engelman, K. \& Portnoy, B. (1970) Circ. Res. 26, 53-57.

7. Da Prada, M. \& Zurcher, G. (1976) Life Sci. 19, 1161-1174.

8. Hallman, H., Farnebo, L. O., Hamberger, B. \& Jonsson, G. (1978) Life Sci. 23, 1049-1052.

9. Brown, M. J. \& Jenner, D. A. (1981) Clin. Sci. 61, 591-598.

10. Hjemdahl, P., Daleskog, M. \& Kahan, T. (1979) Life Sci. 25, $131-138$

11. Miura, Y., Campese, V., De Quattro, V. \& Meijer, D. (1977), J. Lab. Clin. Med. 89, 421-427.

12. Axelrod, J. \& Tomchick, R. (1958) J. Biol. Chem. 233, 702-705.
Dr. Enrico Agabiti-Rosei

Cattedra di Clinica Medica Generale Università c/o I Divisione Medicina

Spedali Civili

I-25100 Brescia 


$$
\text { 。 }
$$

